\title{
CONFERENCIA
}

\section{TRASTORNO DE LOS SONIDOS DEL HABLA - TSH: Cómo el diagnóstico basado en evidencia influye en la intervención}

\author{
Irene Queiroz Marchesan
}

El diagnóstico basado en evidencias influencia en la intervención, una vez que es realizado a partir de investigaciones que comprueben la eficiencia y eficacia de aquello que se propone. Siendo así, en el caso aquí propuesto, el primer paso es saber cuales son los trastornos del habla. A partir de ahí conocer lo que los causan. Para obtener esa respuesta es fundamental, antes de todo, saber anatomía y fisiología de las estructuras que producen el habla. También es importantísimo saber como evaluar, clasificar y reconocer la importancia que los trastornos pueda tener para el paciente. Reflexionemos antes de pensar en investigar algo, necesitamos conocer con profundidad el tema del cual pretendemos discutir. Ser investigador, sin duda, es un verdadero arte; tenemos que lidiar inicialmente con las revisiones sistemáticas sobre el tema, para saber lo que ya fue escrito sobre aquello que se quiere investigar, en seguida tenemos que categorizar y clasificar los textos leídos, además de discutirlos con los que se quiere comprobar. Sumándose a esto, muchas veces, análisis estadísticos serán importantes para dar mayor credibilidad a lo que estamos trayendo al público.

Para encontrar la mejor evidencia de lo que se hace, o se pretende hacer, se debe inicialmente buscar una revisión sistemática, o por lo menos, evidencias basadas en las directrices de la práctica clínica. Existe una dificultad bastante grande para que los profesionales del área de la salud encuentren revisiones sistemáticas sobre un determinado asunto y se mantengan actualizados. Muchas publicaciones en el área de la salud surgen diariamente, no solo en periódicos con y sin revisión de pares, sino también en libros, periódicos y revistas desconocidas, así como en la internet. Para el clínico, le resulta difícil leer todas las investigaciones recientes e incluso conseguir separar lo que es considerado científico, de opiniones o experiencias clínicas diversas. De esa forma, muchas veces el profesional actúa de acuerdo a su propia experiencia acumulada durante su elaboración, apoyado en lecturas que no siempre son basados en experimentos científicos. Así mismo, desde mi punto de vista, los mejores investigadores deberían ser los mejores clínicos, pues el clínico sabe muy bien lo que es la realidad, ya que el la vive diariamente en su consultorio u hospitales.

Las investigaciones consideradas de excelencia en terapia son los ensayos clínicos, aleatorios, ciegos, con control de placebo, que tengan un dibujo paralelo y se calculen si los resultados serán importantes para el paciente. Las medidas utilizadas para evaluar la evidencia para la terapia incluyen el índice de ocurrencia del evento, reducción de los sesgos, número de sujetos necesarios para el tratamiento, posibilidades y proporciones. Aunque se sabe que no todas las definiciones que puedan proponer como ideal sea aceptado universalmente, los principios de lo que se considera como ideal son valiosos para los diagnósticos y tratamientos.

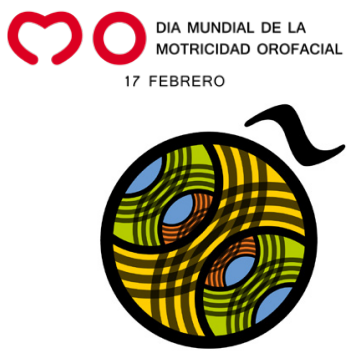

7. Doutorado em Educação pela UNICAMP; Mestre em Distúrbios da Comunicação pela PUC -SP; Especialista em Motricidade Orofacial Título 01/1996; Fonoaudióloga Clínica desde 1978. Atual Presidente da Sociedade Brasileira de Fonoaudiologia Gestão 2014-2016; Publicações de livros, capítulos de livros e artigos na área da Motricidade Orofacial (MO). 